University of Nebraska - Lincoln

DigitalCommons@University of Nebraska - Lincoln

1981

\title{
Simultaneous X-Ray and Optical Observations of AN Ursae Majoris during a Low State
}

\author{
Paula Szkody \\ Einstein Observatory \\ Edward G. Schmidt \\ University of Nebraska at Lincoln, eschmidt1@unl.edu \\ Lidia Crosa \\ Einstein Observatory \\ Robert Schommer \\ Kitt Peak National Observatory
}

Follow this and additional works at: https://digitalcommons.unl.edu/physicsschmidt

Part of the Physics Commons

Szkody, Paula; Schmidt, Edward G.; Crosa, Lidia; and Schommer, Robert, "Simultaneous X-Ray and Optical Observations of AN Ursae Majoris during a Low State" (1981). Edward Schmidt Publications. 46. https://digitalcommons.unl.edu/physicsschmidt/46

This Article is brought to you for free and open access by the Research Papers in Physics and Astronomy at DigitalCommons@University of Nebraska - Lincoln. It has been accepted for inclusion in Edward Schmidt Publications by an authorized administrator of DigitalCommons@University of Nebraska - Lincoln. 
The Astrophysical Journal, 246:223-230, 1981 May 15

(c) 1981. The American Astronomical Society. All rights reserved. Printed in U.S.A.

\author{
SIMULTANEOUS X-RAY AND OPTICAL OBSERVATIONS \\ OF AN URSAE MAJORIS DURING A LOW STATE \\ Paula SzKody ${ }^{1,2,3,4}$, Edward SchmidT ${ }^{2,5}$, Lidia Crosa ${ }^{1,3}$, \\ AND ROBERT SCHOMMER 2,6 \\ Received 1980 August 27; accepted 1980 December 1
}

\begin{abstract}
X-ray observations throughout the orbital cycle of AN UMa during its low state were conducted with the Einstein Observatory simultaneously with ground-based optical $U B V$ photometry and spectroscopy. The observed low X-ray flux of $7 \times 10^{-13} \mathrm{ergs} \mathrm{cm}^{-2} \mathrm{~s}^{-1}$ is a factor of 40 below the high-state X-ray flux, and $F_{x} / F_{\text {opt }}$ is about a factor of 50 lower than for AM Her and 2A 0311-227. There is marginal evidence for an X-ray eclipse coincident with an optical minimum and for increased X-ray flux at magnetic phase 0.4 which does not correlate with any optical continuum variation but may be related to increased hydrogen and helium line emission fluxes.
\end{abstract}

Subject headings: stars: individual - X-rays: binaries

\section{INTRODUCTION}

Krzeminski and Serkowski (1977) found AN UMa to be a member of the AM Her class of high polarization variables. They determined that its circular polarization has a range from -9 to $-35 \%$ in the $B$ filter throughout the 115-minute orbital cycle (Tapia 1977), with ranges of -12 to $-31 \%$ in the $U$ filter and -5 to $-25 \%$ in the $V$ filter. In contrast to the sharp linear polarization spike present in AM Her (Tapia 1977), AN UMa has a broad spike of 0.2 phase width (Krzeminski and Serkowski 1977). Optical photometry (Mumford 1977; Downes and Urbanski 1978; Szkody 1979) shows a large amount of variability in the shape of the light curve. In general, the $U, B$, and $V$ light curves all show a broad minimum at the phase of the most negative excursions of the circular polarization, while maximum light occurs at the phase of the polarization spike. High and low optical states exist between a $V$ range of 14 th and 17th magnitude (Meinunger 1976). Spectroscopy (Schneider and Young 1980) shows prominent $\mathrm{H}$ and $\mathrm{He}$ emission lines with phase-dependent variations and a weak, sharp component which lags the broad by about 0.15 phase.

During a high optical state in 1975 November, AN UMa was detected as a soft X-ray source (Hearn and Marshall 1979) with a $0.1-0.4 \mathrm{keV}$ flux of $2.8 \times 10^{-11}$ ergs $\mathrm{cm}^{-2} \mathrm{~s}^{-1}$ which was modulated with the orbital

\footnotetext{
'Guest Investigator, Einstein Observatory.

${ }^{2}$ Guest Investigator, Kitt Peak National Observatory, operated by AURA, Inc., under contract with the National Science Foundation.

${ }^{3}$ Astronomy Department, University of Washington.

${ }^{4}$ Astronomy Department, University of California, Los Angeles.

${ }^{5}$ Physics and Astronomy Department, University of Nebraska.

${ }^{6}$ Astronomy Department, California Institute of Technology.
}

cycle. The X-ray minimum occurred $180^{\circ}$ away from the linear polarization spike.

In order to investigate further this orbital dependence and to correlate it with existing photometric and spectroscopic observations to aid in determining the geometry of the system, simultaneous X-ray and optical observations were made throughout several cycles of AN UMa. The system was in a low optical state during the observations.

\section{OBSERVATIONS}

The X-ray observations were made with the Imaging Proportional Counter (IPC) on the Einstein Observatory (see Giaconni et al. 1979 for a description of the satellite and instrumentation), yielding measurements over an available energy range of $0.1-4 \mathrm{keV}$ (the exact energies varied somewhat on each night because of varying gains). The first observations were scheduled for 1979 May 19 and 20 with coordinated optical photometry at the $1.3 \mathrm{~m}$ Kitt Peak National Observatory (KPNO) telescope and spectroscopy at Lowell Observatory. However, last minute changes imposed by Goddard resulted in a very short X-ray observation (about 30 minutes on each of the two nights), so the entire observation had to be rescheduled for the next X-ray window in 1979 November (which was actually beneficial since the May weather at both KPNO and Lowell was generally uncooperative). A few representative $U B V$ light curves were obtained on 1979 May 17 and 21 by P.S. with the three-channel photometer and on 1979 May 30 by R.S. with the Mark II photometer. These measurements determined that AN UMa was in a low optical state $(V \sim 16.5)$. Figure 1 shows typical $U B V$ light curves obtained at this time. 

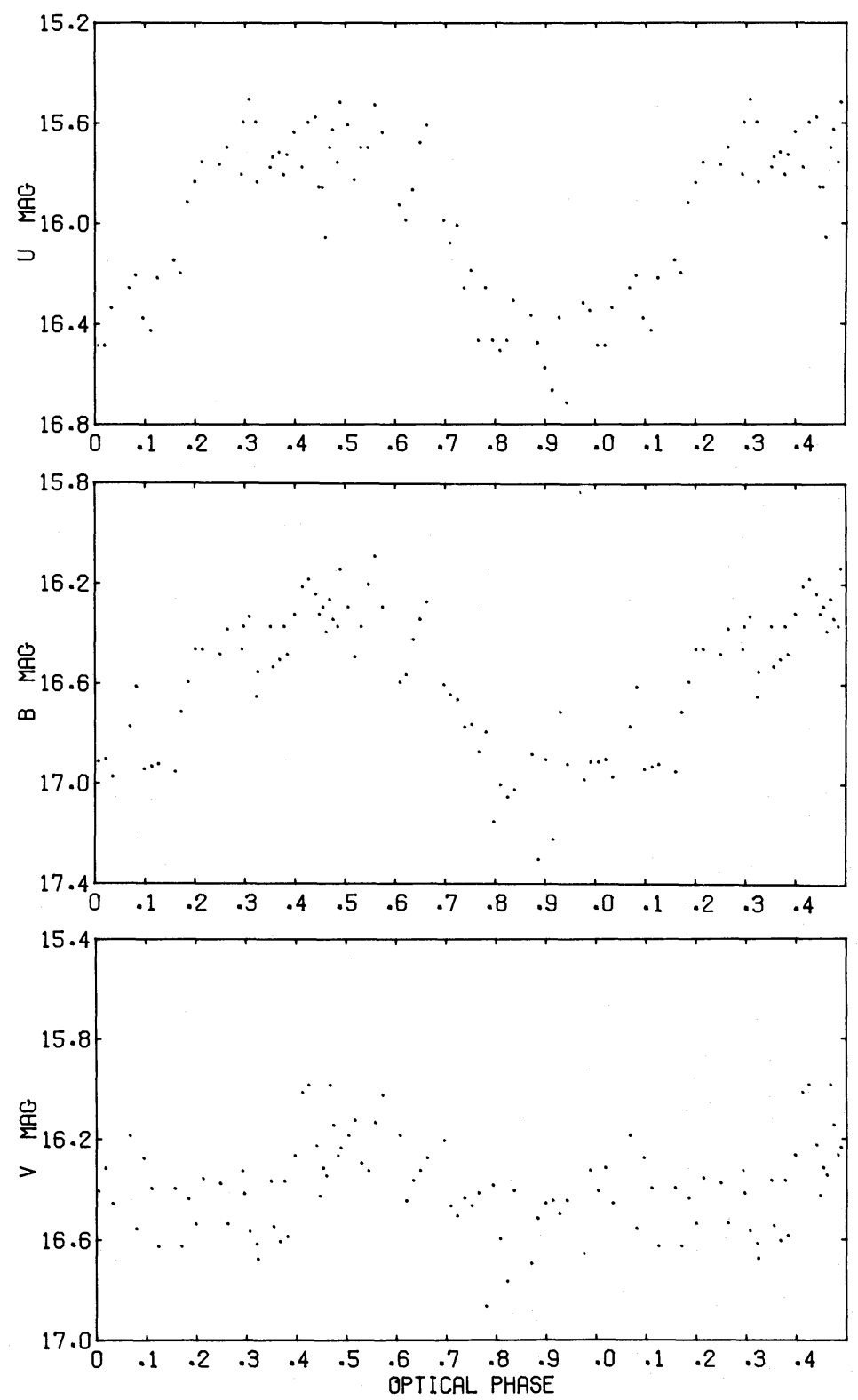

FIG. 1.- The $U B V$ light curves obtained on 1979 May 30 UT. Each point represents the sum of 2 five-second integrations with uncertainties of $\pm 0.1 \mathrm{mag}$.

On 1979 November 20 and 21 the complete orbital coverage by Einstein was obtained during a 3-hour pointing on each of the two nights. Coordinated $U B V$ photometry on both nights was accomplished by E.S. on the $1.3 \mathrm{~m}$ telescope at KPNO using the Mark II photometer which cycled between filters. One-minute integration times were used (about 4 minutes per cycle), giving statistical uncertainties less than $\sim 0.05$ mag (except for $V$ the night of November 20 when the uncertainty was up to $0.11 \mathrm{mag}$ because of a noisy phototube). In addition, on November 20, A. Wilson and J. Ulvestad used the Intensified Image Dissector Scanner (IIDS) system on the KPNO $2.1 \mathrm{~m}$ telescope to obtain two sets of spectra from $3800 \AA-7100 \AA$ with a resolution of about $10 \AA$. Each set consisted of 16 1-minute integrations which were summed to obtain a usable signal-to-noise. Figures 2 and 3 show the coordinated optical and X-ray light curves for November 20 and 21 , respectively. The time intervals of the spectroscopy are marked in Figure 2, while the two sets of 


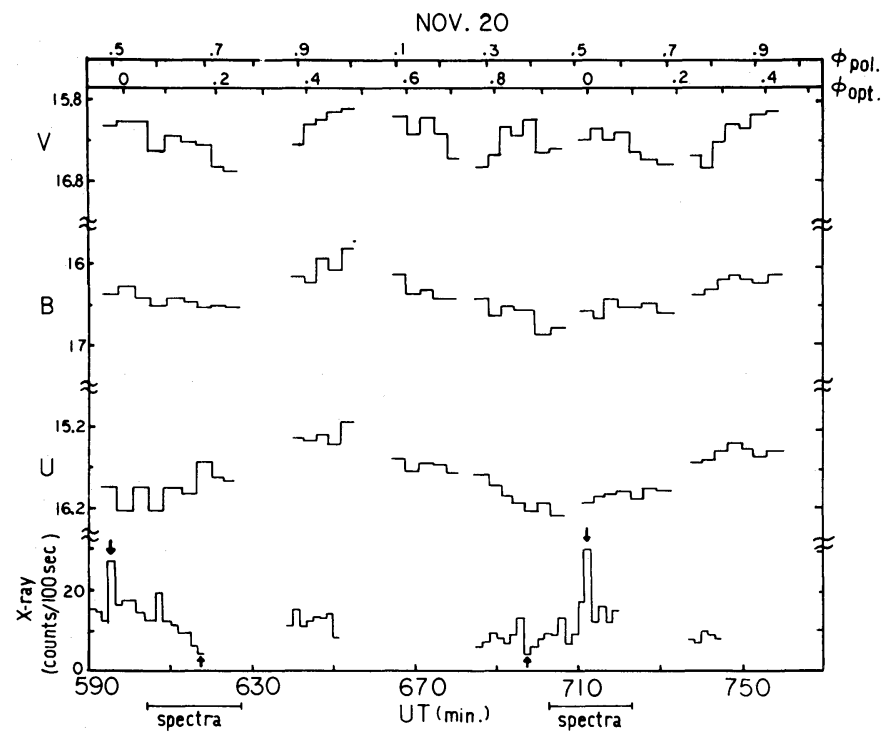

FIG. 2. - The $U B V$ magnitudes and IPC binned counts for 1979 November 20 UT. The arrows represent the intervals which are $\geq 3 \sigma$ deviations from the mean. The times during which IIDS spectra were obtained are marked.

summed spectra are shown in Figure 4. The phasing used is:

$$
\begin{aligned}
\text { optical minimum }= & \text { HJD 2,442,502.285 } \\
& +0.07975307,
\end{aligned}
$$

polarization maximum $=$ HJD $2,443,191.7879$

$$
+0.07975307 \text {, }
$$

where the period is that reported by Schneider and
Young (1980), the polarization spike phase is from Krzeminski and Serkowski (1977), and the optical minimum phasing is from Mumford (1976).

An infrared $(J, H, K)$ light curve was obtained by P.S. 1 month after the X-ray observation (1979 December 31 and 1980 January 2) with the KPNO $4 \mathrm{~m}$ telescope and Blue Toad InSb system. Sixteen measurements at each filter with a signal-to-noise ratio of $10 / 1$ were obtained throughout the orbital cycle.

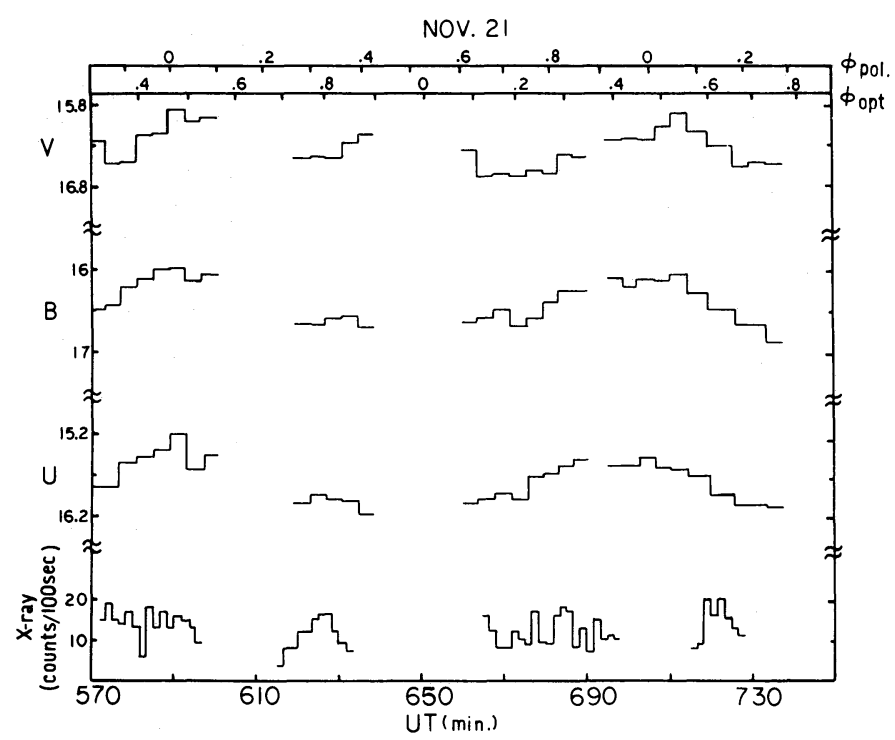

FIG. 3. - The $U B V$ magnitudes and IPC binned counts for 1979 November 21 UT. 
FIRST SET
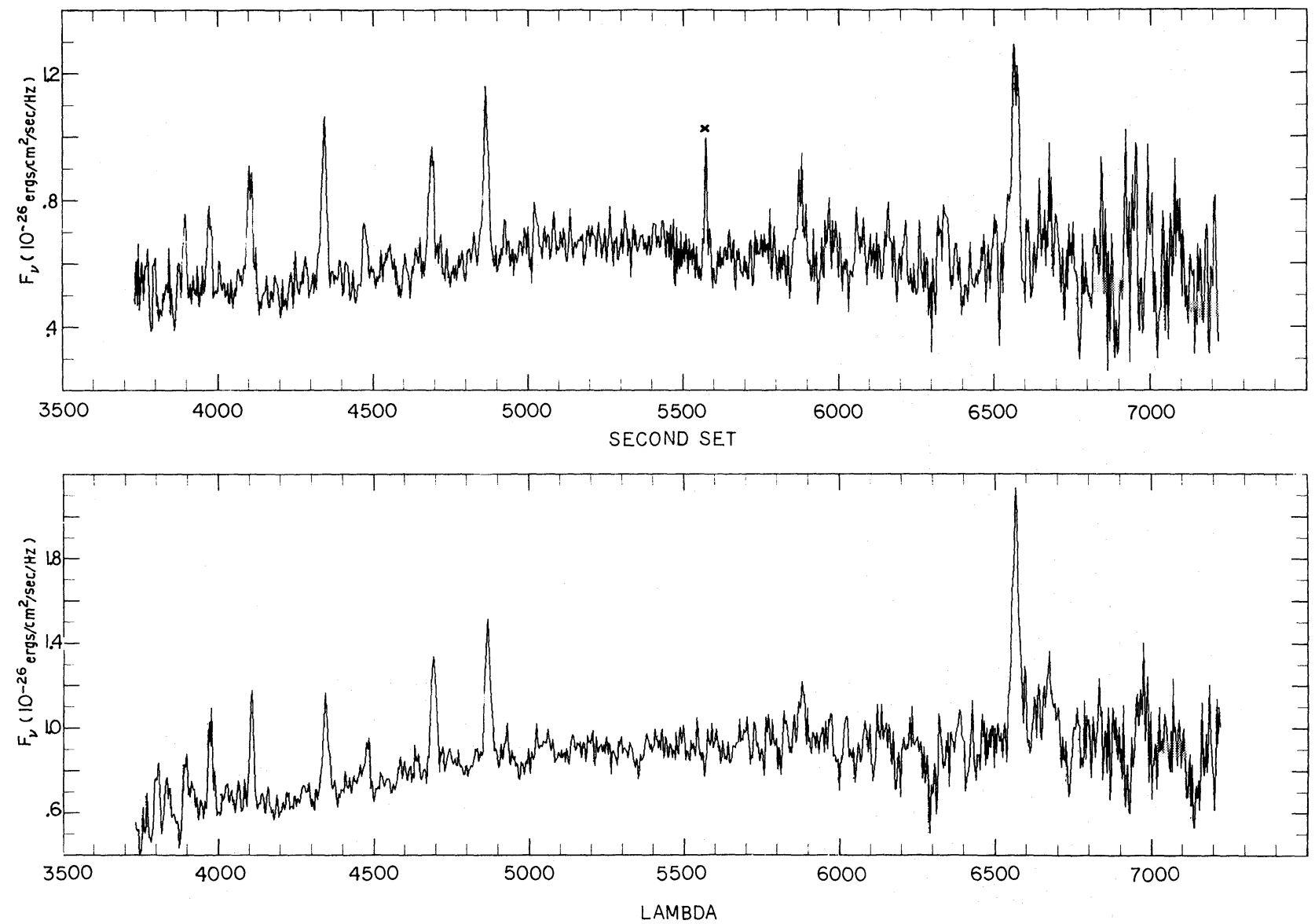

FIG. 4. - The two sets of spectra obtained by A. Wilson and J. Ulvstad during the time intervals shown in Fig. 2. Each set is the sum of 16 one-minute integrations. The $\times$ marks the position of a night-sky line.

\section{DISCUSSION}

\section{a) Total X-Ray Flux}

During the 1979 May and November observations, the mean count rate with the IPC from all energy channels was 0.12 counts $\mathrm{s}^{-1}$. Transforming this to a flux is difficult since a good calibration of the IPC does not exist. However, an estimate of the hardness of the source may be made by comparing the count rate in the various overlapping channels. Because changes occur in the gain from one day to the next, which alters the energy in each channel slightly, this comparison was made separately for each night's data. The values for several energies during the observation of November 21 are listed in Table 1, with similar values obtained for the rest of the observations. It can be seen that most of the flux is from the soft X-ray band $(E \leq 0.5 \mathrm{keV})$. This is the same as the result found by Hearn and Marshall

TABLE 1

X-RAy DATA FOR 1979 November 21

\begin{tabular}{|c|c|c|c|}
\hline$E(\mathrm{keV})$ & Counts s ${ }^{-1}$ & Background & $\begin{array}{c}F \times 10^{-13} \\
\left(\text { ergs cm } \mathrm{cm}^{-2} \mathrm{~s}^{-1}\right)\end{array}$ \\
\hline $0.1-0.5$ & 0.073 & $5 \times 10^{-5}$ & 6.5 \\
\hline $0.5-1.0$ & 0.028 & $1 \times 10^{-5}$ & 0.002 \\
\hline $1.0-2.0 \ldots$ & 0.015 & $1 \times 10^{-5}$ & $<0.001$ \\
\hline $2.0-4.0 \quad \ldots$ & 0.008 & $2 \times 10^{-5}$ & $<0.001$ \\
\hline
\end{tabular}


TABLE 2

COMPaRison OF High aNd Low STATES

\begin{tabular}{|c|c|c|}
\hline$V$ & Total Flux (ergs $\left.\mathrm{cm}^{-2} \mathrm{~s}^{-1}\right)$ & Hardness Ratio \\
\hline $14.5 \ldots$ & $2.8 \times 10^{-11 \mathrm{a}}$ & 0.25 \\
\hline $16.3 \ldots$ & $7.2 \times 10^{-13}$ & 0.75 \\
\hline
\end{tabular}

${ }^{\mathrm{a}}$ From Hearn and Marshall 1979.

(1979) for AN UMa during the high optical state. Their data were consistent with a mean $k T_{\mathrm{BB}} \sim 40 \mathrm{eV}$ and $N_{\mathrm{H}} \sim 2 \times 10^{20} \mathrm{~cm}^{-2}$. If we assume these same parameters at the low state and fold them through the instrument response, we obtain the fluxes listed in Table 1 as a function of energy and a total mean flux of $7.2 \times 10^{-3}$ ergs $\mathrm{cm}^{-2} \mathrm{~s}^{-1}$ over all channels. The hardness ratio $E(0.5-4.5 \mathrm{keV}) / E(0.1-0.5 \mathrm{keV})=0.75$.

A comparison with Hearn and Marshall (1979) data from the high state is made in Table 2. It is apparent that, while the optical light decreases by a factor of 6 from high to low state, the X-ray flux appears to decrease by a factor of roughly 40 with only a slight increase in hardness.

\section{b) The X-Ray Light Curve}

The low X-ray flux at the low optical state makes it difficult to determine orbital dependence. The data was divided into $100 \mathrm{~s}$ bins on each of the 4 days. Out of a total of 152 bins, there are only 4 which show peaks $\geq 3 \sigma$ from the mean. However, 3 out of the 4 occur at the same phase ( 0.5 polarization phase), and Hearn and Marshall's (1979) X-ray curve also shows a spike near this same time (their magnetic phase 0.7 becomes phase 0.6 with the period of Schneider and Young 1980). It is possible that we cannot trust the phasing from 1975 through 1979 since phase drifts are expected (Joss, Katz, and Rappaport 1979). However, the phasing in AM Her from the observed optical and X-ray light curves appears to be stable over a 5-year period (Szkody et al. 1980), and the optical $B$ minima in the light curves of AN UMa with the given ephemeris match the observations of Mumford (1977) in 1976 and 1977 and Downes and Urbanski (1978) in 1978. Thus, we think it is reasonable to compare our X-ray data with that of Hearn and Marshall (1979).

The Hearn and Marshall (1979) X-ray data taken during the high optical state also show a minimum in the X-ray light curve about 0.1 phase preceding the spike. During the period of our low optical state data, there is also some marginal evidence for this minimum. Out of the 152 bins, there are 8 with deviations $\geq 3 \sigma$ below the mean, and 5 out of the 8 occur during polarization phase $0.4-0.5$. Thus, there is some evidence that the shape and features of the X-ray light curve are preserved during the change from high to low state, while only the overall intensity is lowered.

\section{c) Optical and Infrared Data}

The overall characteristics of the optical light curves obtained in 1979 May and November and the IR curve from 1979 December may be summarized:

1. A broad minimum of 1 mag depth exists in $U$ and $B$ centered near optical phase 0 (polarization phase 0.53 ).

2. A shallower, filled-in minimum at this phase occurs in $V$ with lowest points ( $0.7 \mathrm{mag}$ depth) at optical phases 0.25 and 0.75 - see Figure 2 .

3. Maximum light in $U, B$, and $V$ occurs at optical phase 0.5 (polarization phase 0 ).

4. The optical minimum (optical phase 0 ) is red ( $B-$ $V \sim 0.3$ ) while the optical maximum (phase 0.5 ) is bluer $(B-V \sim-0.1)$.

5. No apparent orbital variation occurs in the IR (to within $\pm 0.1 \mathrm{mag}$ ) with mean values over the orbit of $J=15.6, H=15.2$, and $K=15.0$. These values are consistent with those obtained in 1978 (Szkody and Capps 1980), and the colors $(V-K=1.0)$ imply that the source of the IR is not a late-type secondary (as is apparent in VV Pup at minimum) nor a cyclotron IR peak (as in AM Her).

There is no evidence in the $U B V$ light curves for any sharp spike near optical phase 0 which would compare with the spike seen in Hearn and Marshall's (1979) data and the largest deviations from the mean in our X-ray data. However, comparison of the line fluxes from the two sets of spectra (set 1 after the spike phase and set 2 including the spike phase-see Table 3) shows larger fluxes in the set with the possible spike. Schneider and Young (1980) report a sharp component of the line is only present during polarization phases $0.7-0.1$, so that, at the phase of the possible X-ray spike, we are only dealing with the broad component which is believed to originate from near the accretion column.

The ratios $\mathrm{H} \alpha / \mathrm{H} \beta=1.2,1.3$ and $\mathrm{H} \gamma / \mathrm{H} \beta=1.4,1.1$ for the two sets of spectra reveal a steeper Balmer decrement than for AM Her (ratios 0.75 and 1Stockman et al. 1977) and 2A 0311-227 (ratios 0.4 and 1.2-Griffiths et al. 1979). The ratios for AN UMa are consistent with the lower Balmer lines being optically thick and imply $N_{e} \sim 10^{14} \mathrm{~cm}^{-3}$ and $T_{e} \sim 1-2 \times 10^{4} \mathrm{~K}$ for the emission region (Drake and Ulrich 1980).

TABLE 3

LiNe FluXES $\left(\times 10^{-14}\right.$ ergs $\left.\mathrm{cm}^{-2} \mathrm{~s}^{-1}\right)$

\begin{tabular}{lccc}
\hline \hline Line & 1st Set & 2nd Set & Set 2/Set 1 \\
\hline $\mathrm{H} \alpha \ldots \ldots \ldots$ & 1.4 & 2.1 & 1.5 \\
$\mathrm{H} \beta \ldots \ldots \ldots$ & 1.2 & 1.6 & 1.3 \\
$\mathrm{H} \gamma \ldots \ldots \ldots$ & 1.7 & 1.8 & 1.1 \\
$\mathrm{He} 4686 \ldots$ & 1.1 & 1.4 & 1.3 \\
$\mathrm{He} 4471 \ldots$ & 0.6 & 0.8 & 1.4 \\
$\mathrm{He} 5876 \ldots$ & 0.6 & 0.7 & 1.2 \\
\hline
\end{tabular}




\section{d) Model Implications}

The proposed models for AM Her and VV Pup encompass those with one active pole (Chanmugam and Wagner 1977) or two active poles (King and Lasota 1979; Liebert and Stockman 1979). Even quadrupoles have been suggested for 2A 0311-227 (Allen, Ward, and Wright 1980). In AM Her, the presence of both positive and negative circular polarization, the offset of the primary $V$ minimum by half an orbit from the $\mathrm{X}$-ray minimum, and the lack of correlation between the X-ray and optical flickering (Szkody et al. 1980) all argue for a two-pole system with the X-rays originating from one pole and the optical light from the dominant pole. In VV Pup, changes in the circular polarization argue for a switch between accretion at one pole during very low optical states to two-pole accretion at high optical states (Liebert and Stockman 1979).

In the case of AN UMa, there is not enough optical and circular polarization data at high and low states to determine if there are changes from a one-pole to a two-pole system. At the low optical state, the circular polarization is always negative (Krzeminski and Serkowski 1977), and the most negative polarization comes at the same phase as the single minimum in the blue light curve and at the same phase as the X-ray minimum present at the high state (optical phase 0 and polarization phase 0.53 ). This argues for a system with one active pole.

Schneider and Young (1980) suggest a one-pole model for the AN UMa system which is consistent with the observations. The inclination of the system to the line of sight is $35^{\circ}$. The angle of the dipole axis to the $z$ axis is $50^{\circ}$ and to the $x$ axis is $20^{\circ}$ (see Fig. 5 for a drawing with this geometry plus the location of the various optical and X-ray events). Thus, at polarization phase 0 the pole is nearly perpendicular to the observer, while at optical minimum (polarization phase 0.5 , and inferior conjunction of the red star) the pole is at an angle of $18^{\circ}$ to the observer. In this type of model, the optical and $\mathrm{X}$-ray minimum must be caused by optical depth effects when looking down into the column while maximum light occurs when the column is at right angles to the line of sight. The line emission originates from both the accretion column and the heated region of the secondary.

An explanation of the X-ray spike present at the high optical state and possibly also at the low will require a peculiar geometry in the given model. From Figure 5, it is apparent that the column is viewed at a low angle at the phase of the spike. If the soft X-rays originate from the heated surface of the white dwarf, the presence of the spike implies a change in the opacity of the middle of the column which affects only the X-rays and line emission areas. It would be useful to see if this is apparent in the UV, but the faintness of the system is beyond the capabilities of $I U E$ to obtain the necessary

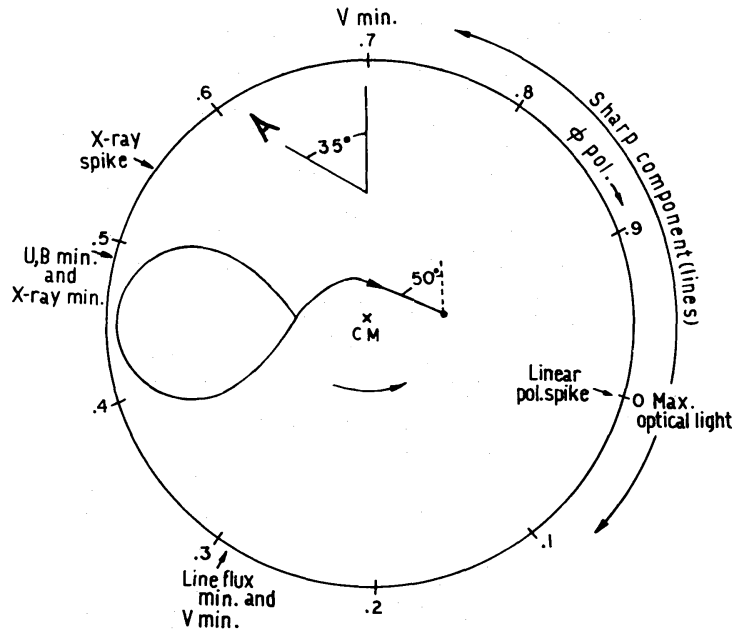

FIG. 5. - A schematic model for the system following the notation of Schneider and Young (1980), where the system is viewed in the $x-y$ plane and the rotation axis is about the $z$ axis. The $x$ axis joins the secondary and the white dwarf. In the third dimension, there is a rotation of the dipole axis by $20^{\circ}$ with respect to the $x$ axis. The polarization phases are marked together with the various occurrences seen by the observer.

time resolution, and so we must await a more sensitive instrument.

According to the model of Lamb and Masters (1979), hard X-rays originate in a shock in the lower region of the accretion column. Half of the X-rays escape outward, and the other half heat the white dwarf surface which then emits as a blackbody in the UV and soft $\mathrm{X}$-ray region. In this type of picture $\left(L_{\mathrm{BB}} \approx L_{\mathrm{brem}}+L_{\mathrm{cyc}}\right.$ (where $L_{\mathrm{BB}}$ is the blackbody luminosity, $L_{\mathrm{brem}}$ is the bremsstrahlung luminosity, and $L_{\text {cyc }}$ is the cyclotron luminosity). However, study of the UV flux in AM Her by Raymond et al. (1979) has shown that the strong UV cyclotron emission that was predicted by the theory is not observed and $L_{\mathrm{BB}} \gg L_{\text {brem }}+L_{\text {cyc }}$. With the limited observations available for AN UMa, it is difficult to determine if we are faced with the same problem. Hartmann and Raymond (1980) have obtained short wavelength IUE spectra of AN UMa in 1979 October while it was at a low state $(V \sim 16 \mathrm{mag})$. While the signal strength was low and variability was evident between the two exposures, there is marginal evidence for a $\nu^{2}$ continuum flux distribution. Since the exposures were 2 and 4 hours, there is no way to sort out orbital variability (J. Raymond, private communication). The flux at $1500 \AA$ is $\sim 3 \times 10^{-15} \mathrm{ergs} \mathrm{cm}^{-2} \mathrm{~s}^{-1}$. Assuming the $\mathrm{X}$-ray flux to be the same as during our observations, then this UV flux is greater than $\sim 500$ times the flux that can be expected as the tail end of a $25-40 \mathrm{eV}$ soft $\mathrm{X}$-ray blackbody (energies within the range reported for the high state of AN UMa by Hearn and Marshall 1979). To determine if this flux is consistent with the predicted cyclotron emission, we would need to know 
the value of the electron temperature (there is no $20 \mathrm{keV}$ component yet detected from AN UMa).

For $T_{\mathrm{BB}} \sim 25-40 \mathrm{eV}$ and a distance of $100 \mathrm{pc}$, the observed $0.1-0.5 \mathrm{keV}$ flux gives $L_{\mathrm{BB}} \sim 10^{31-32} \mathrm{ergs} \mathrm{s}^{-1}$. Since there is no observed bremsstrahlung component, $L_{\text {brem }}$ will be $\ll L_{\mathrm{BB}}$, and assuming the $\nu^{2}$ continuum flux distribution in the UV is cyclotron emission up to a cutoff wavelength of $1000 \AA$ (the cutoff for AM Her) will give $L_{\mathrm{cyc}} \sim 0.1 L_{\mathrm{BB}}$. It is conceivable that the $\nu^{2} \mathrm{UV}$ component is produced by a hot white dwarf, as in AM Her at its low state (J. Raymond and L. Hartmann, private communication). Then $L_{\text {cyc }}$ would be even lower than the above estimate. Thus, within the limits of the sparse data available, we are led to the conclusion that, as in AM Her, $L_{\mathrm{BB}}>L_{\text {brem }}+L_{\text {cyc }}$, and the interpretation of AN UMa with the Lamb and Masters (1979) model will be difficult.

From Figure 6, it is apparent that the optical and IR fluxes are not the continuation of the $\nu^{2}$ flux distribution from the UV. This optical continuum could be similar to the $F_{\nu} \propto \nu^{-1}$ component seen in AM Her.

The flux distribution from X-ray to IR is shown in Figure 6 , where the $\mathrm{X}$-ray and optical points are the average over the orbital cycle on 1979 November 21, the IR points the average over the cycle on 1979 December 31 and January 2, and the UV points from the 1979 October data of Hartmann and Raymond (1980). This distribution may be compared to the X-ray-IR distribution of AM Her (Stockman et al. 1977) and the opticalIR distribution of VV Pup (Szkody and Capps 1980). The flux distribution of both AM Her and VV Pup peaks at about $1 \mu \mathrm{m}$, which appears to be the result of cyclotron emission in AM Her and the secondary star in VV Pup. In AN UMa, the peak is near the $V$ region of the spectrum.

The X-ray-optical fluxes for AN UMa show a lower ratio than for AM Her and 2A $0311-227$, and this ratio changes as a function of state. While the optical flux of AN UMa during a low state is about a factor of 6 less than in AM Her during a low state in 1976 May (Priedhorsky and Krzeminski 1978), the soft X-ray flux is less by about a factor of 300 (Hearn and Richardson 1977). The system $2 \mathrm{~A} 0311-227$ has a $L_{x} / L_{\text {opt }}$ ratio which is the same as AM Her (Griffiths et al. 1979).

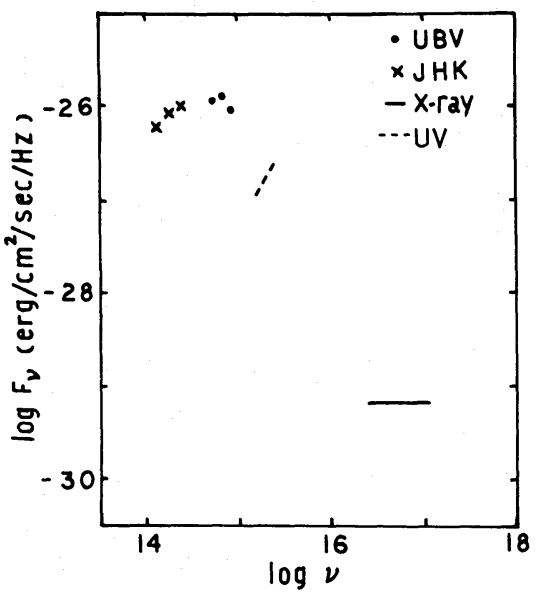

FIG. 6. - The flux distribution versus frequency for AN UMa from the soft X-ray through the IR. The UV points are from Hartmann and Raymond (1980).

Between the low and the high optical state, AN UMa brightened optically by a factor of 8 while the soft X-ray brightness increased by about a factor of 50. In AM Her, $F_{x} / F_{\text {opt }}$ remains about the same during the different states (these flux differences are summarized in Table 4). This means that part of the large difference in $\mathrm{X}$-ray emission between AN UMa and AM Her may result from a different change in parameters in going from high to low states. Mumford (1977) reports that the optical minimum at the high state is narrower and deeper than at the low state. No information exists as to the polarization or spectroscopic changes from low to high. It is conceivable that the known optical and X-ray differences could be ascribed to changes in the mass transfer rate between low and high states which can change the strength of the shock and, thus, the component from the heated white dwarf, as well as the opacity and width of the accretion column. Integrating the optical-IR flux distribution and using a distance of 100 pc gives a luminosity of $10^{31}$ ergs $\mathrm{s}^{-1}$ (in contrast to $10^{33}$ for AM Her-Stockman et al. 1977). Assuming the luminosity is due to accretion gives $\dot{M} \sim 10^{-12} M_{\odot}$ $\mathrm{yr}^{-1}$, a factor of 100 less than in AM Her (Stockman et al. 1977). If this low luminosity is not a distance effect,

TABLE 4

Optical and X-Ray Fluxes during Low and High States

\begin{tabular}{|c|c|c|c|c|}
\hline Object & State & $V$ & $F_{\mathrm{V}}\left(\operatorname{ergs~} \mathrm{cm}^{-2} \mathrm{~s}^{-1} \mathrm{~Hz}^{-1}\right)$ & $F_{x}\left(\operatorname{ergs~cm} \mathrm{cm}^{-2} \mathrm{~s}^{-1} \mathrm{~Hz}^{-1}\right)$ \\
\hline \multirow[t]{2}{*}{ AM Her .... } & Low & 14.5 & $6.3 \times 10^{-26}$ & $2.1 \times 10^{-27 a}$ \\
\hline & High & 12.8 & $3.0 \times 10^{-25}$ & $5.9 \times 10^{-27 b}$ \\
\hline \multirow{2}{*}{ AN UMa ... } & Low & 16.3 & $1.2 \times 10^{-26}$ & $6.7 \times 10^{-30}$ \\
\hline & High & 14 & $1.0 \times 10^{-25}$ & $3.9 \times 10^{-28 c}$ \\
\hline
\end{tabular}

\footnotetext{
${ }^{a}$ Hearn and Richardson 1977

b Tuohy et al. 1978

${ }^{\mathrm{c}}$ Hearn and Marshall 1979
} 
the lower accretion rate probably results in the lower $\mathrm{X}$-ray fluxes.

The authors gratefully acknowledge Dr. A. Wilson and J. Ulvestad for obtaining the spectra used in this paper; Dr. N. White for attempting to thwart the clouds; Drs. G. Fabbiano and F. Cordova for help with the
Einstein reductions; J. Barnes and Dr. D. Hayes for assistance with the KPNO photometers; Dr. J. Raymond for useful comments on the UV spectrum; and Drs. J. Katz and B. Margon for comments on the manuscript. This research was supported in part by NASA grant NAS8-33337.

\section{REFERENCES}

Allen, D. A., Ward, M. J., and Wright, A. E. 1980, preprint. Chanmugam, G., and Wagner, R. 1977, Ap. J. (Letters), 213, L13. Downes, R. A., and Urbanski, J. L. 1978, Pub. A.S.P., 90, 458.

Drake, S. A., and Ulrich, R. K. 1980, preprint.

Giaconni, R., et al. 1979, Ap.J., 230, 540

Griffiths, R. E., Ward, M. J., Blades, J. C., Wilson, A. S., Chaisson, Lola, and Johnston, M. D. 1979, Ap. J. (Letters), 232, L27.

Hartmann, L., and Raymond, J. 1980, to be published in "The Universe at Ultraviolet Wavelengths-The First 2 Years of IUE" (NASA).

Hearn, D. R., and Marshall, F. J. 1979, Ap. J. (Letters), 232, L21. Hearn, D. R., and Richardson, J. A. 1977, Ap. J. (Letters), 213, L115.

Joss, P. C., Katz, J. I., and Rappaport, S. A. 1979, Ap. J., 230, 176.

King, A. R., and Lasota, J. P. 1979, M.N.R.A.S., 188, 653

Krzeminski, W., and Serkowski, K. 1977, Ap.J. (Letters), 216, L45.

Lamb, D. Q., and Masters, A. R. 1979, Ap.J. (Letters), 234, L117.

Liebert, J., and Stockman, H. S. 1979, Ap. J., 229, 652.
Meinunger, L. 1976, Inf. Bull. Var. Stars No. 1168.

Mumford, G. 1976, Inf. Bull. Var. Stars No. 1133. 1977, Sky and Tel., 54, 194

Priedhorsky, W. C., and Krzeminski, W. 1978, Ap. J., 219, 547.

Raymond, J. C., Black, J. H., Davis, R. J., Dupree, A. K., Gursky, H., Hartmann, L., and Matilsky, T. A. 1979, Ap. J. (Letters), 230, L95.

Schneider, D., and Young, P. J. 1980, preprint.

Stockman, H. S., Schmidt, G. D., Angel, J. R. P., Liebert, J., Tapia, S., and Beaver, E. A. 1977, Ap. J., 217, 815.

Szkody, P. 1979, in IAU Colloquium 5i, Stellar Turbulence, ed.

D. F. Gray (New York: Springer-Verlag), p. 324

Szkody, P., and Capps, R. 1980, A. J., 85, 882

Szkody, P., Córdova, F. A., Tuohy, I. R., Stockman, H. S., Angel, J. R. P., and Wisniewski, W. 1980, Ap. J., 241, 1070.

Tapia, S. 1977, IAU Circ., No. 3049.

Tuohy, I. R., Lamb, F. K., Garmire, G. P., and Mason, K. O. 1978, Ap.J. (Letters), 226, L17.

Lidia Crosa and Paula Szkody: Astronomy Department, University of Washington, Seattle, WA 98195

EDWARD Schmidt: Physics and Astronomy Department, University of Nebraska, Lincoln, NE 68588

Robert Schommer: Institute of Astronomy, University of Cambridge, Madingley Road, Cambridge CB3 0HA England 